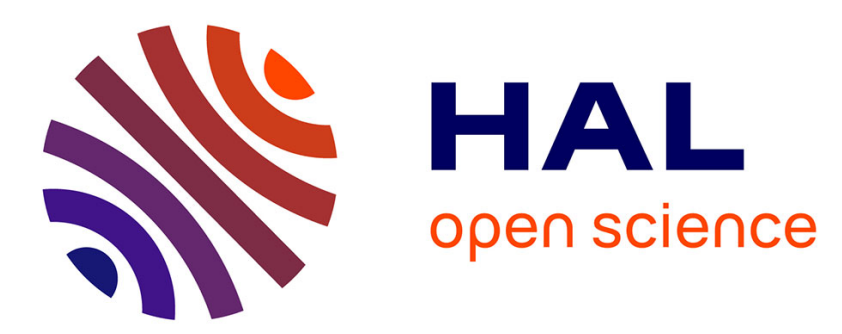

\title{
Control of plasma properties in capacitively coupled oxygen discharges via the electrical asymmetry effect
}

E Schüngel, Q-Z Zhang, S Iwashita, J Schulze, L-J Hou, Y-N Wang, U

Czarnetzki

\section{- To cite this version:}

E Schüngel, Q-Z Zhang, S Iwashita, J Schulze, L-J Hou, et al.. Control of plasma properties in capacitively coupled oxygen discharges via the electrical asymmetry effect. Journal of Physics D: Applied Physics, 2011, 44 (28), pp.285205. 10.1088/0022-3727/44/28/285205 . hal-00633995

\section{HAL Id: hal-00633995 https://hal.science/hal-00633995}

Submitted on 20 Oct 2011

HAL is a multi-disciplinary open access archive for the deposit and dissemination of scientific research documents, whether they are published or not. The documents may come from teaching and research institutions in France or abroad, or from public or private research centers.
L'archive ouverte pluridisciplinaire HAL, est destinée au dépôt et à la diffusion de documents scientifiques de niveau recherche, publiés ou non, émanant des établissements d'enseignement et de recherche français ou étrangers, des laboratoires publics ou privés. 


\title{
Control of plasma properties in capacitively coupled oxygen discharges via the Electrical Asymmetry Effect
}

\author{
E Schüngel $^{1}$, Q-Z Zhang ${ }^{2}$, S Iwashita ${ }^{1}$, J Schulze ${ }^{1,3}$, L-J Hou ${ }^{4}$, \\ Y-N Wang ${ }^{2}$ and U Czarnetzki ${ }^{1}$
}

1 Insitute for Plasma and Atomic Physics, Ruhr-University Bochum, 44780 Bochum, Germany

2 School of Physics and Optoelectronic Technology, Dalian University of Technology, China

3 Research Institute for Solid State Physics and Optics, Hungarian Academy of Sciences, 1525 Budapest POB 49, Hungary

4 Max Planck Institute for Extraterrestrial Physics, 85748 Garching, Germany

E-mail: Edmund.Schuengel@ep5.rub.de

\begin{abstract}
By using a combined experimental, numerical and analytical approach, we investigate the control of plasma properties via the Electrical Asymmetry Effect (EAE) in a capacitively coupled oxygen discharge. In particular, we present the first experimental investigation of the EAE in electronegative discharges. A dual-frequency voltage source of $13.56 \mathrm{MHz}$ and $27.12 \mathrm{MHz}$ is applied to the powered electrode and the discharge symmetry is controlled by adjusting the phase angle $\theta$ between the two harmonics. It is found that the bulk position and density profiles of positive ions, negative ions, and electrons have a clear dependence on $\theta$, while the peak densities and the electronegativity stay rather constant, largely due to the fact that the time averaged power absorption by electrons is almost independent of $\theta$. This indicates that the ion flux towards the powered electrode remains almost constant. Meanwhile, the dc self-bias and, consequently, the sheath widths and potential profile can be effectively tuned by varying $\theta$. This enables a flexible control of the ion bombarding energy at the electrode. Therefore, our work proves the effectiveness of the EAE to realize separate control of ion flux and ion energy in electronegative discharges. At low pressure, the strength of resonance oscillations, which are found in the current of asymmetric discharges, can be controlled with $\theta$.
\end{abstract}

PACS numbers: 52.80.Pi, 52.50.-b, 52.27.Cm, 52.35.Fp 


\section{Introduction}

For many years, capacitively coupled radio frequency (CCRF) discharges have been an interesting subject of research due to a variety of physical and chemical processes therein, and particularly the vast area of applications, such as the thin film deposition and etching as well as many other surface processings in semiconductor and panel display industries. In most of these applications, separate control of the ion flux and the ion bombarding energy onto the processing surface is of great importance for manufacturing efficiency and quality control [1]. The concept of dual frequency discharges, i.e., driving the plasma with two substantially different radio frequencies, had, therefore, been introduced as a promising way to achieve this separate control [2-8]. However, it is known that the coupling of both frequencies [9-19] and the effect of secondary electrons $[8,20-22]$ places a strong limitation to realize this goal. A rather new approach was recently proposed to control the ion properties via the Electrical Asymmetry Effect (EAE) [23-37]: By applying a voltage waveform, that contains a fundamental frequency and (at least) one even harmonic, the symmetry of the discharge can be changed by tuning the phase angle between the driving harmonics. This in turn allows controling basic properties of the plasma, such as the dc self-bias, the position of the plasma bulk, and the ion energy at both electrodes while maintaining an almost constant ion flux.

Earlier studies of both experiments and numerical simulations had shown that the EAE is very effective in realizing separate control of ion properties in electropositive discharges, such as discharges in argon. However, in most applications a mixture of gases revealing complex chemical and physical features is employed. And most of these gases are electronegative. For example, a gas mixture of $\mathrm{O}_{2}$ and $\mathrm{CF}_{4}$ is used for silicon etching $[38,39]$. And with a small amount of additions of silane and oxygen, a helium discharge can be used to deposit $\mathrm{SiO}_{2}$ [40]. In both examples and many others negative ions play a very important role in controlling the chemical and physical properties of the plasmas. And many of the plasma properties are different from those in electropositive discharges. It is, therefore, worth studying thoroughly the EAE in electronegative plasmas. This has been our motivation of conducting a combined experimental, numerical and analytical investigation of CCRF discharges in pure oxygen. Oxygen discharges, being one of the simplest electronegative plasma system, provide an interesting scenario for theoretical modeling (see for example [41-50, 52] and references therein) and experimental diagnostics [49, 50, 52-57] of electronegative plasmas. In contrast to the previous work of the EAE in oxygen discharges with only numerical simulation [36], our complementary approach here certainly promises a more comprehensive description of the discharge properties.

This article is organized as follows. In Sec. 2, we describe the details of our experimental setup, numerical simulation and the analytical model of CCRF discharges. Results are presented in Sec. 3, which is divided into three subsections. First, we show the influence of the EAE on the density profiles of ions and electrons, the peak plasma density, the electronegativity, and the time averaged power absorption. Second, we 
show the way to control the electrical symmetry and, accordingly, the dc self-bias. And third, the self-excitation of plasma series resonance oscillations is discussed. Finally, conclusions are drawn in Sec. 4.

\section{Description of Experiment, Simulation, and Model}

\subsection{Experiment}

The experimental setup is similar to the one that has been used and described in previous studies [27]. Therefore, only a brief description is given here. The discharge is set up in a modified GEC (Gaseous Electronics Conference) reference cell. A pure oxygen plasma is ignited between the lower powered electrode and the upper grounded electrode, both of which have a radius of $5.0 \mathrm{~cm}$. The electrode gap is $2.5 \mathrm{~cm}$. The plasma is confined radially between the electrodes by a glass cylinder. Therefore, the electrode configuration is symmetric. However, the discharge is not perfectly symmetric, since the side confinement of the plasma by the glass cylinder effectively enhances the grounded surface area. This leads to a certain geometrical asymmetry, depending on the discharge conditions [58].

Oxygen gas is injected through four gas inlets into the chamber, but outside the plasma region. The applied voltage waveform consists of a fundamental frequency component with frequency $f$ and its second harmonic:

$$
\bar{\phi}_{\sim}(t)=\phi_{0} \frac{1}{2}[\cos (2 \pi f t+\theta)+\cos (4 \pi f t)],
$$

where $f=13.56 \mathrm{MHz}$ is the fundamental frequency used in both experiment and simulation. $\theta$ is the fixed, but adjustable phase angle between the driving frequencies. In the experiment, it is generated and matched by two synchronized function generators, amplifiers, and matching boxes. An electrical filter is used to prevent one frequency component from penetrating into the other ones amplifier. The voltage between the filter and the powered electrode is measured by a high voltage probe. The amplitudes of the applied harmonics and the relative phase between them are calibrated with respect to the values on top of the powered electrode, if the chamber is vented. The time averaged voltage yields the dc self-bias. The plasma current flowing to the grounded electrode is measured time resolved by a self-excited electron resonance spectroscopy (SEERS) sensor. Squaring this current yields a measure for the power absorbed by the electrons.

\subsection{Simulation}

We employ here a standard $1 \mathrm{~d} 3 \mathrm{v}$ (one dimensional in space and three dimensional in velocity) Particle-in-Cell/Monte Carlo Collision (PIC/MCC) method [59] to simulate DF-CCRF discharges in pure oxygen in a gap of $2.5 \mathrm{~cm}$ (corresponding to our experimental conditions). The waveform of the driving voltage on the powered electrode (at $x=0$ ) is given by Eq. 1, while the other electrode is grounded. The amplitudes of 
the driving voltage are again taken from corresponding experimental measurements. As is expected, by changing $\theta$, the absolute values of the positive and negative extremes in the waveform and the voltages across the two sheaths will change accordingly [24], and this leads to a dc self-bias at the powered electrode. The self-bias voltage is determined in the simulation in an iterative way to ensure that the net current to the two electrodes in one low frequency RF cycle is zero [60].

In the MCC part of the simulation, we follow the standard procedure for oxygen [61], except (i) the volume production and surface loss processes of the lowest lying metastable state of the oxygen molecule $\mathrm{O}_{2}\left(a^{1} \triangle_{g}\right)$; and (ii) the recombination processes with only reaction rates $\kappa$ available. The former is beyond the scope of a one-dimensional model and we thus assume a constant density of $\mathrm{O}_{2}\left(a^{1} \triangle_{g}\right)$ in the whole chamber of $10 \%$ of $\mathrm{O}_{2}$ density (corresponding to the data of Gudmundsson et al [46] at the chamber center) as Bronold et al did in [48]. For the collisions, which only have rate constant $\kappa$ available, we adopt Nanbu's method [62] based on Direct Simulation Monte Carlo.

An explicit scheme is used in the simulations and the subcycling technique [63] is adopted for ions. We use 700 uniform cells with about 200 particles per cell and run the simulation for typically $4000-5000$ low frequency periods to converge. We take the ion-induced secondary electron-emission coefficient $\gamma_{i}=0.12$. Two different pressures, namely, $10 \mathrm{~Pa}$ and $100 \mathrm{~Pa}$ are considered. More details of the simulation, such as collision processes considered in the simulation and processing method can be found in [36].

\subsection{Model}

The analytical model used to describe capacitive discharges was first introduced in [24]. We apply a voltage waveform according to Eq. 1 to the powered electrode. The amplitude of the low (1f) and high (2f) frequency component is $\phi_{0} / 2$, which altogether gives the total amplitude $\phi_{0}$.

In the voltage balance of a capacitive discharge, we assume the well known quadratic charge voltage dependence [31] for the sheaths. The net positive total charge within the discharge volume, i.e. inside the non-quasineutral sheaths, is almost constant as a function of time. The time dependent total charge oscillations can be expected to be even smaller in the electronegative oxygen discharge than in argon [31], because the fraction of electrons in the total amount of charges inside the plasma is smaller. Indeed, in the simulations it only changes by about $\pm 1 \%$. Having made these assumptions, the voltage balance is

$$
\bar{\phi}_{\sim}(t)+\bar{\eta}=-q^{2}(t)+\varepsilon\left(q_{t}-q(t)\right)^{2}-2 \beta^{2}\left(\ddot{q}+\nu_{m} \dot{q}\right) .
$$

Here, the applied voltage $\bar{\phi}_{\sim}(t)$ and dc self-bias $\bar{\eta}$ are normalized by the applied voltage amplitude $\phi_{0}$. The net charge within the powered electrode sheath, $q(t)$, and the total charge, $q_{t}$, are normalized by the maximum charge in the powered electrode sheath, $Q_{0}$, i.e. $0 \leq q(t) \leq 1$. Here, $Q_{0} \approx A_{p} \sqrt{2 e \varepsilon_{0} \bar{n}_{s p} \phi_{0}}$ is a normalization constant with $\bar{n}_{s p}$ the mean ion density in the powered electrode sheath [34]. 
$\varepsilon_{0}$ is the vacuum permittivity and $e$ the elementary charge. The bulk part is obtained from the eletron momentum balance equation; it consists of a resistive part and an inductive component representing the electron inertia. A dot represents differentiation with respect to time. The bulk factor $\beta=\sqrt{\left(\varepsilon_{0} m_{e} A_{p} L_{b}\right) /\left(e^{2} \bar{n}_{b} \bar{A}_{b} s_{\text {max }, p}\right)}$ depends on the ratio of the surface area of the powered electrode, $A_{p}$, to an effective bulk surface area, $\bar{A}_{b}$, and the ratio of the bulk length, $L_{b}$, to the maximum extension of the powered electrode sheath, $s_{\max , p} \cdot m_{e}$ is the electron mass and $\bar{n}_{b}$ an effective electron density in the bulk. Typically, $\beta \ll 1$. Therefore, we neglect the voltage drop across the plasma bulk region in the following to obtain time averaged expressions. Even though it might become important in discharges with high electronegativity due to the reduced electron density in the plasma bulk, we justify this assumption by a time averaged bulk voltage of $\leq 1 \%$ resulting from PIC/MCC simulations. However, it will be important to take it into account for the analytical description of the plasma series resonance, which is self-excited in asymmetric discharges at low gas pressures.

Eq. 2 directly leads to analytical expressions for the normalized dc self-bias, $\bar{\eta}$, and the normalized total charge within the discharge, $q_{t}[24,34]$ :

$$
\begin{aligned}
\bar{\eta} & =-\frac{\bar{\phi}_{\sim, \max }+\varepsilon \bar{\phi}_{\sim, \min }}{1+\varepsilon}, \\
q_{t} & =\sqrt{\frac{\bar{\phi}_{\sim, \max }-\bar{\phi}_{\sim, \min }}{1+\varepsilon}} .
\end{aligned}
$$

Therefore, $\bar{\eta}$ can be adjusted by changing $\bar{\phi}_{\sim, \max }$ and $\bar{\phi}_{\sim, \min }$, i.e. by tuning $\theta$. The symmetry parameter

$$
\varepsilon=\left|\frac{\phi_{s g, \max }}{\phi_{s p, \max }}\right| \approx\left(\frac{A_{p}}{A_{g}}\right)^{2} \frac{\bar{n}_{s p}}{\bar{n}_{s g}}
$$

is defined as the ratio of the absolute values of the maximum voltage drop across the powered $\left(\phi_{s p, \text { max }}\right)$ and grounded $\left(\phi_{s g, \text { max }}\right)$ electrode sheath. In the case of a geometrically symmetric discharge, $A_{p}=A_{g}$, $\varepsilon$ depends to a good approximation only on the ratio of the mean positive ion densities in the powered $\bar{n}_{s p}$ and grounded $\bar{n}_{s g}$ electrode sheath [24].

The maximum sheath extensions, $s_{\max , p}$ and $s_{\max , g}$, are calculated based on the Brinkmann sheath criterion [64]

$$
\int_{0}^{s(t)} n_{-}(x, t) d x=\int_{s(t)}^{d / 2}\left(\left\langle\bar{n}_{+}(x)\right\rangle-n_{-}(x, t)\right) d x
$$

at the time of maximum extension of the respective sheath, which are $t\left(s_{\max , p}\right)=$ $t\left(\phi_{\sim, \min }\right)$ and $t\left(s_{\max , g}\right)=t\left(\phi_{\sim, \max }\right)$. Here, $\left\langle n_{+}(x)\right\rangle$ is the time averaged spatially resolved density of positive ions $\left(O_{2}^{+}\right)$and the negative charge density $n_{-}(x, t)$ is the sum of the time dependent electron density, $n_{e}(x, t)$, and the negative ion density, $\left\langle n_{O_{-}}(x)\right\rangle$.

Under the assumption of a negligible bulk contribution, the charge voltage balance (Eq. 2) can easily be solved for the time dependent charge in the powered electrode sheath, $q(t)$. The first derivative of $q(t)$ with respect to time, $\dot{q}(t)$, is proportional 
to the electron conducting current density: $j_{e}(t)=-\left(Q_{0} / A_{p}\right) \dot{q}(t)$. Again, $Q_{0}$ is the normalization constant of $q(t)$ introduced above. This directly leads to the power absorbed by the plasma electrons, $P_{e}(t)$ [32]. We find $P_{e}(t)=\left[j_{e}^{2}(t) / \sigma\right] A_{p} L_{b}$ by using the bulk length $L_{b}$ and conductivity $\sigma \propto \bar{n}_{b}$ [1] for a given pressure. The time averaged value of $P_{e}$,

$$
\left\langle P_{e}\right\rangle=P_{e, 0}\left\langle\dot{q}^{2}\right\rangle,
$$

is of particular interest, since the constancy of $\left\langle P_{e}\right\rangle$ as a function of $\theta$ apparently is the reason for the bulk densities and the ion fluxes to remain almost constant, independently of $\theta[24,32]$. Here, $P_{e, 0}=2 \phi_{0} \varepsilon_{0} e \bar{n}_{s p} A_{p} L_{b} / \sigma$ depends on the ratio of the positive ion density in the powered electrode sheath and the electron density in the plasma bulk. The analytical approximation of $\left\langle P_{e}\right\rangle$, which holds under all conditions investigated here, has been studied in detail before [32].

\section{Results}

\section{1. control of the density profiles}

Figure 1 (a)-(c) shows the spatial distributions of positive ions, negative ions, and electrons for different phase angles resulting from PIC/MCC simulations operated at a low pressure. The density profiles of both ion species show one distinct maximum located inside the plasma bulk, whereas the density profile of the plasma electrons is flat in the bulk region. This is caused by the rather long mean free path of the electrons $\left(\lambda_{m f p} \approx 7 \mathrm{~mm} \lesssim L_{b} \approx 10 \mathrm{~mm}\right.$ at $\left.10 \mathrm{~Pa}\right)$. Therefore, fast electrons, which are accelerated in the sheath regions [36, 52-55, 65-77], ionize the background gas within the entire plasma bulk region and the common diffusion profiles are obtained for the positive ions. The negative ions are confined within this region; due to their low kinetic energy, they cannot penetrate into the sheath regions, where the time averaged potential is much lower than the plasma potential in the bulk. Similar density profiles are found in capacitively coupled single frequency oxygen discharges by different authors $[41,42,44,49]$.

The maximum extension of the sheaths at the powered and grounded electrode shown in Fig. 1 (d) are varied and the bulk region is moved by tuning the phase angle $\theta$ from $0^{\circ}$ to $180^{\circ}$. In this way, the density profiles of the charged species can be shifted away from the discharge center towards the powered or grounded electrode. However, this shift is quite small under these conditions compared to the scenario of even lower pressures discussed in argon [28] and oxygen [36] before. In addition, it is found that the bulk length $L_{b}$, which we define as the difference between the electrode gap and the sum of the maximum sheath extension at both electrodes,

$$
L_{b}=d-\left(s_{\max , p}+s_{\max , g}\right) \text {, }
$$

is slightly modulated as a function of $\theta$. It is smaller in the symmetric cases, i.e. at $\theta \approx 45^{\circ}$ and $\theta \approx 135^{\circ}$, compared to the asymmetric cases obtained at the phase angles 

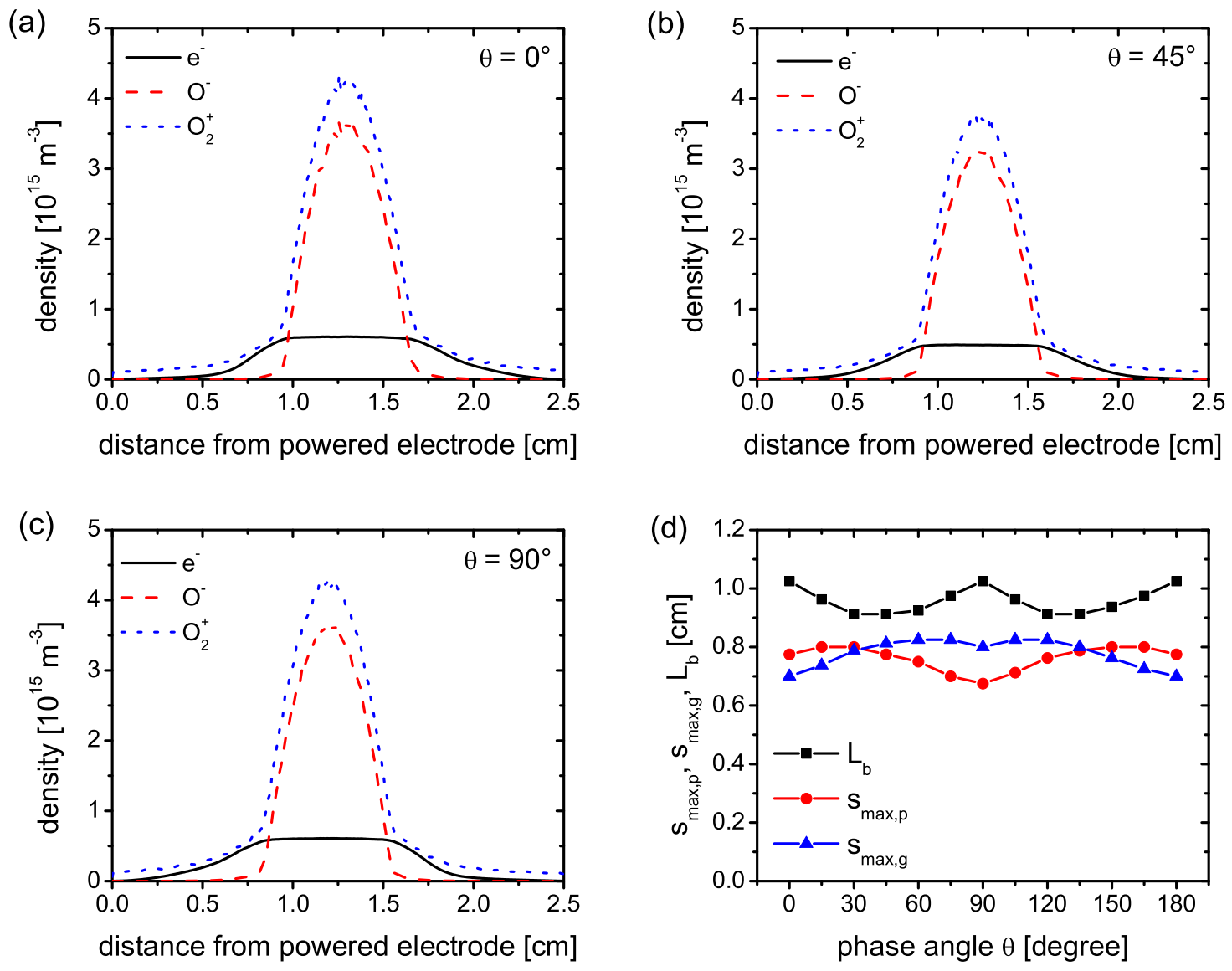

Figure 1. Spatial distribution of positive ions, negative ions, and electrons between the powered electrode and the grounded electrode resulting from PIC/MCC simulations: (a) $\theta=0^{\circ}$, (b) $\theta=45^{\circ}$, (c) $\theta=90^{\circ}$. (d) Length of the plasma bulk and maximum extension of the sheath at the powered and grounded electrode. $\left(10 \mathrm{~Pa}, \phi_{0}=150 \mathrm{~V}\right.$, $\mathrm{d}=2.5 \mathrm{~cm})$

$\theta \approx 0^{\circ}, \theta \approx 90^{\circ}$, and $\theta \approx 180^{\circ}$, respectively. This also affects the maximum electron density and the peak densities of both the positive and the negative ions, which are found to be about $20 \%$ (electrons) and $10 \%$ (ions) smaller in the symmetric cases (Fig. 1 (b)) compared to the asymmetric ones (Fig. 1 (a) and (c)).

At relatively high pressure, the spatial density distributions look completely different. As can be seen in Fig. 2 (a) - (c), the density of both ion species shows two peaks located close to the maximum sheath extension of the powered and grounded electrode. Of course, the sheath widths shown in figure 2 (d) generally are much smaller compared to the low pressure scenario. The position of the density peaks remains almost unchanged as a function of the phase angle $\theta$. However, the peak density itself shows a strong dependence on the discharge symmetry, which is adjusted via $\theta$. This might give the opportunity to spatially control the source of radicals, which are important in surface processing. Between these peaks a concave density profile is found, which is 

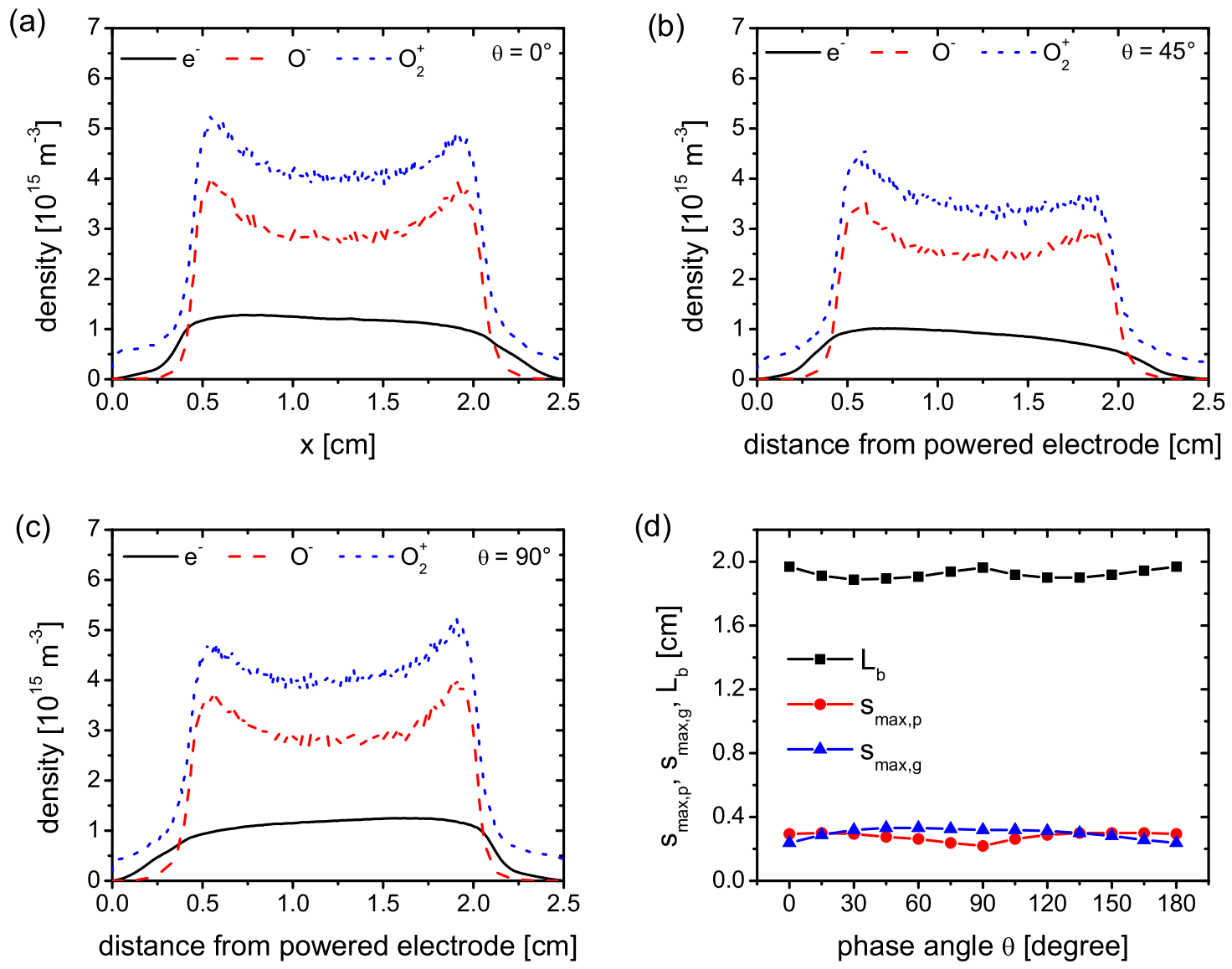

Figure 2. Spatial distribution of positive ions, negative ions, and electrons between the powered electrode and the grounded electrode resulting from PIC/MCC simulations: (a) $\theta=0^{\circ}$, (b) $\theta=45^{\circ}$, (c) $\theta=90^{\circ}$. (d) Length of the plasma bulk and maximum extension of the sheath at the powered and grounded electrode. $\left(100 \mathrm{~Pa}, \phi_{0}=150 \mathrm{~V}\right.$, $\mathrm{d}=2.5 \mathrm{~cm})$

typical in the high pressure regime with neutral detachment being the dominant loss mechanism for negative ions in the discharge volume $[44,45,50]$. The decrease of the density profile between the peaks in the bulk centre might be overestimated, for instance due to the assumption of a spatially constant metastable density. However, a detailed discussion about the formation mechanisms of the peaks in the ion density profiles is beyond the scope of this work. Moreover, the profiles are in good agreement with the results of other groups [41, 43, 44, 49].

In contrast to the low pressure case, the electron mean free path is short $\left(\lambda_{m f p}<\right.$ $1 \mathrm{~mm} \ll L_{b} \approx 17 \mathrm{~mm}$ at $100 \mathrm{~Pa}$ ). Therefore, the production of positive ions is strongly localized in front of the sheaths, where the electrons are accelerated. Starting from these planes, diffusion takes place within the bulk region, leading to the flat ion profiles. Due to this localization of production, the peak density of positive and negative ions strongly interacts with the electron heating within the respective sheath. As will be seen later, 
this will affect the symmetry parameter and the control range of the dc self-bias.

As it has been observed in oxygen discharges [78-80] as well as in other electronegative plasmas [81-84], a double layer may appear in electronegative discharges under certain conditions. The formation of a double layer leads to a peak in the electron density localized in front of the electrode sheaths around the time of sheath collapse. However, one criterion for the formation of a double layer is that the negative ions reasonably contribute to the time resolved total discharge current [80, 84]. Due to the relatively small electronegativity and the low mobility of negative ions compared to the electron mobility, this is not the case under all conditions investigated here. Therefore, we do not observe any double layer structures.

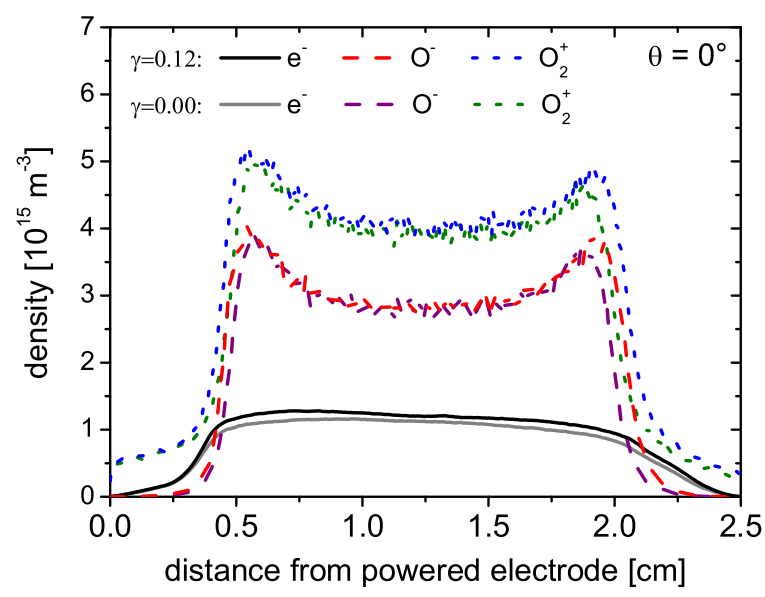

Figure 3. Spatial distribution of positive ions, negative ions, and electrons between the powered and the grounded electrode resulting from PIC/MCC simulations for two different values of the secondary electron emission coefficient: $\gamma=0.12$ and $\gamma=0$. The other discharge conditions are the same as in figure 2. $\left(100 \mathrm{~Pa}, \phi_{0}=150 \mathrm{~V}, \mathrm{~d}=2.5\right.$ $\mathrm{cm})$

In order to estimate the impact of secondary electrons on our simulation results, a comparison between the density profiles obtained in a simulation with $\gamma=0.12$ (secondary electron emission coefficient that is used throughout this article, see Sec. 2.2) and $\gamma=0$ (no secondary electrons released from the electrode surfaces) under otherwise identical discharge conditions is given in figure 3. The total densities are marginally higher in the case including secondary electrons due to the enhanced ionization by fast electrons. However, this does not affect the shape of the density profiles, which are almost the same for both values of $\gamma$. Therefore, all results discussed here are not expected to be very sensitive with respect to the specific choice of $\gamma$.

The electronegativity is, among others, a discharge parameter that is often used to describe the composition of species in oxygen plasmas [47]. It can be defined in two different ways, either by taking the ratio of the peaks of the density distributions or by 


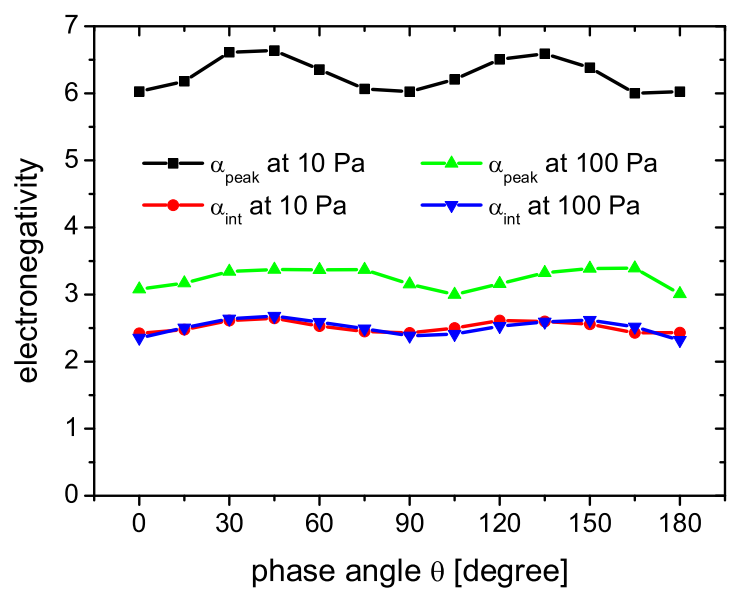

Figure 4. Electronegativity as a function of the phase angle at low and high pressure resulting from PIC/MCC simulations. By definition, $\alpha_{\text {peak }}$ and $\alpha_{\text {int }}$ is the ratio of the peak and integrated density profiles of negative ions and electrons, respectively. $\left(\phi_{0}=150 \mathrm{~V}, \mathrm{~d}=2.5 \mathrm{~cm}\right)$

spatially integrating these distributions [1]:

$$
\begin{aligned}
\alpha_{i n t} & =\frac{\int_{0}^{d} n_{O^{-}}}{\int_{0}^{d} n_{e}}, \\
\alpha_{\text {peak }} & =\frac{n_{O^{-}, \max }}{n_{e, \max }} .
\end{aligned}
$$

Figure 4 shows the electronegativity, $\alpha_{\text {int }}$ and $\alpha_{\text {peak }}$ according to the definitions above, resulting from PIC/MCC simulations at low and high pressure as a function of the phase angle $\theta$. Due to the relatively small change of the densities of ions and electrons as a function of the phase angle (also see Figs. 1 and 2), the electronegativity is almost constant as a function of $\theta$ under all conditions investigated here. In fact, the change in the bulk length depending on the phase angle discussed above leads to the small modulations observed. At low pressure, the peaked shape of the density distribution of $\mathrm{O}^{-}$and the flat distribution of the electron density cause $\alpha_{\text {peak }}$ to be much bigger than $\alpha_{i n t}[47]$. At high pressure, both density profiles are rather flat. Thus, the difference between the electronegativity obtained using Eqs. 9 and 10, respectively, is small. The absolute values determined here are comparable with the results obtained by other authors in single frequency capacitive discharges. [41, 42, 44-46, 50, 55]

From figure 4 we conclude that $\alpha_{\text {peak }}$ decreases with the neutral gas pressure, whereas the spatially averaged electronegativity, $\alpha_{i n t}$, remains almost constant. A similar pressure dependence of $\alpha_{\text {peak }}$ was observed before [41, 42]. Other groups found $\alpha_{\text {int }}$ to increase as a function of pressure in single frequency capacitive discharges [50, 51]. However, a direct comparison of the results is not possible due to the strong dependence of the electronegativity on all discharge parameters, e.g. the applied rf power [44], and the general disparity between these discharge conditions in single and dual frequency 
discharges.
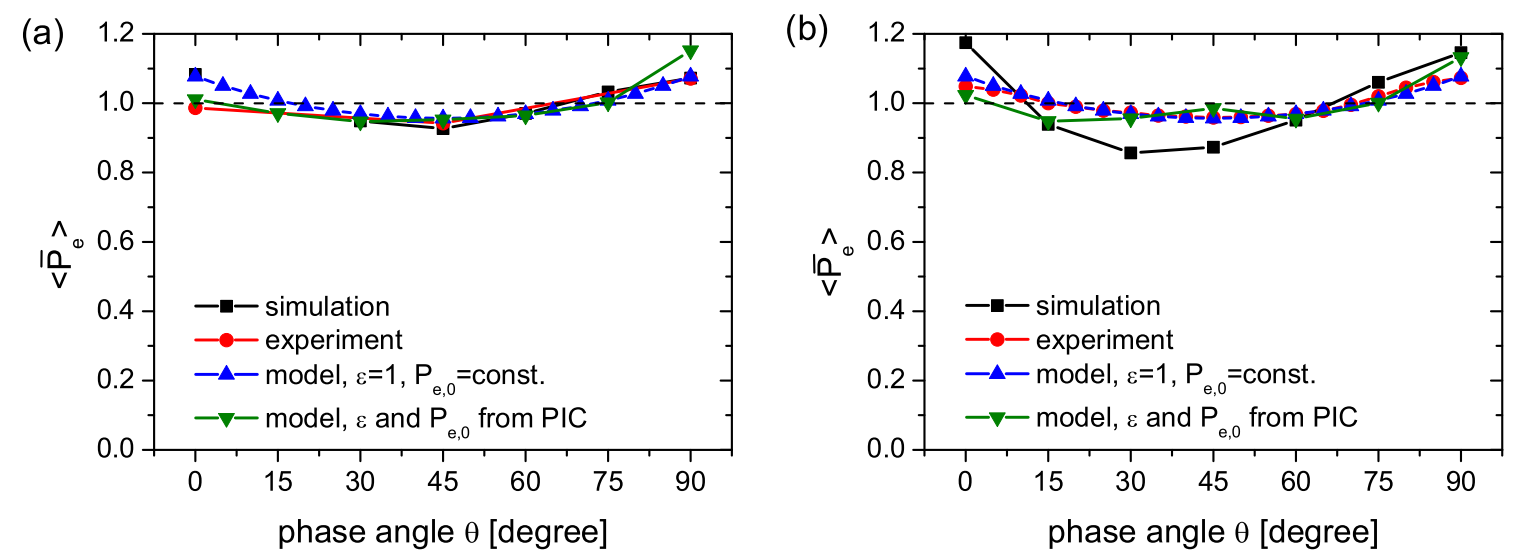

Figure 5. Time averaged power absorbed by the plasma electrons as a function of $\theta$ at low and high pressure resulting from experiment, simulation, and model. $\left\langle P_{e}(\theta)\right\rangle$ is normalized by the mean value for all $\theta$. (a) low pressure (10 Pa), (b) high pressure $(100 \mathrm{~Pa}) .\left(\phi_{0}=150 \mathrm{~V}, \mathrm{~d}=2.5 \mathrm{~cm}\right)$

To explain the reason why the bulk densities and the electronegativity remain constant to a good approximation as a function of $\theta$, it is useful to examine the time averaged power absorbed by the plasma electrons, $\left\langle P_{e}\right\rangle$. Figure 5 shows $\left\langle P_{e}\right\rangle$ as a function of $\theta$ at low and high pressure resulting from experiment, simulation, and model. The period averaged absorbed power is normalized by the mean value for all $\theta$. At both low and high pressure, the mean electron power stays rather constant as a function of $\theta$. A slight decrease is found around $\theta \approx 45^{\circ}$, which is due to the weak dependence of the normalized total charge, $q_{t}$, on $\theta$ [32]. Therefore, the density of positive ions, which are mainly produced by collisions between fast electrons and the neutral background gas molecules, depends only weakly on $\theta$. As a consequence, the flux of positive ions onto the electrodes is approximately constant, independently of $\theta$. This constancy was obtained in Ref. [36] and can be explained here. It should be noted that this argument might fail in other electronegative, e.g. fluorine containing, gas mixtures [8].

Since the analytical model does not include the effect of secondary electrons, the good agreement between all three approaches indicates that the discharge is operated in the $\alpha$-mode, i.e., the power absorption by the plasma electrons is mainly due to sheath expansion heating. This can also be recognized from the rather small effect of secondary electrons on the densities of charges species (see figure 3). In addition, the phase between the applied rf voltage (equation 1) and the rf component of the discharge current appearing later in this manuscript (figures 8 and 10), is approximately out of phase by $90^{\circ}$. By comparing this phase with literature data [85], this also indicates that the discharge burns in the $\alpha$-mode. 

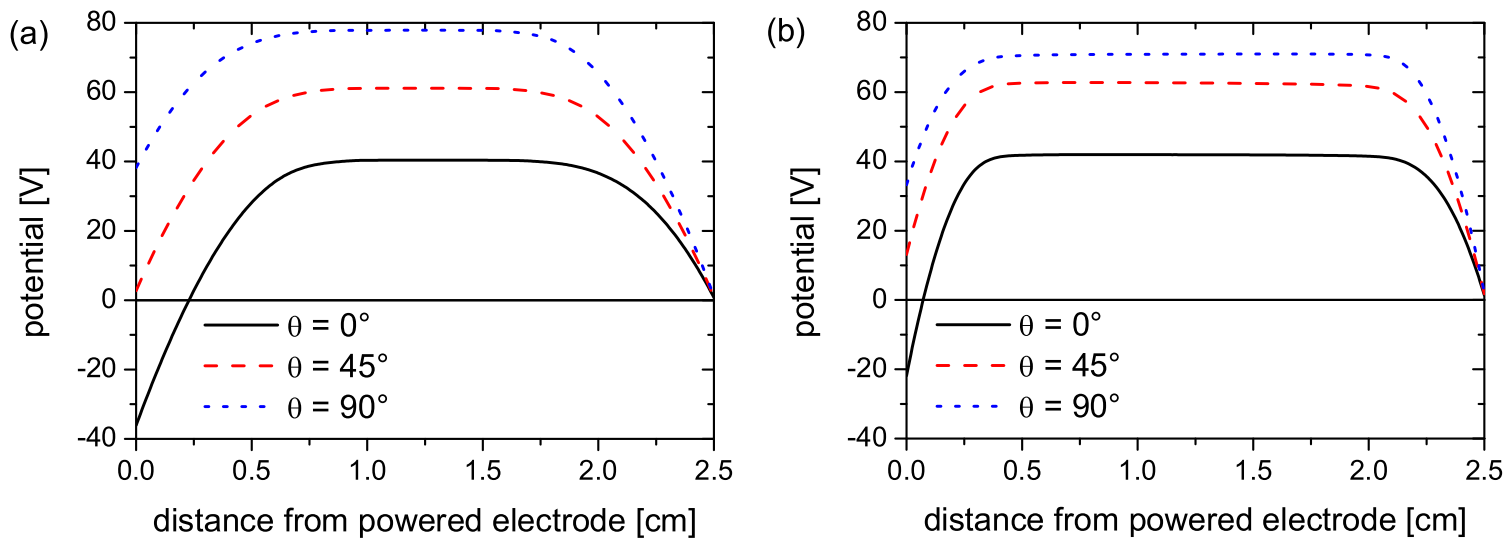

Figure 6. Time averaged potential profile between the powered and grounded electrode for different phase angles resulting from PIC/MCC simulations. (a) low pressure $(10 \mathrm{~Pa}),(\mathrm{b})$ high pressure $(100 \mathrm{~Pa}) .\left(\phi_{0}=150 \mathrm{~V}, \mathrm{~d}=2.5 \mathrm{~cm}\right)$

\section{2. control of the electrical symmetry}

In this section, the control of electrical potentials in capacitively coupled oxygen discharges via the EAE is explained. We concentrate on time averaged potentials, which are important for the ion species due to their relatively low plasma frequency. $[1,2,86]$ Figure 6 shows the time averaged potential profile between the powered and grounded electrode for different phase angles at low and high pressure resulting from $\mathrm{PIC} / \mathrm{MCC}$ simulations. In general, the profile reveals the common shape consisting of a plasma bulk region with a negligible voltage drop confined between the sheaths in front of the powered and grounded electrode [1]. The latter regions show a strong potential decrease. Thus, negative ions cannot reach the electrodes by overcoming the potential barrier and are well confined within the discharge. It can also be seen that the sheath regions are bigger at low pressure [2], which is in good agreement with the data shown in Figs. 1 (d) and 2 (d), respectively.

The control of the mean potential at the powered electrode and of the time averaged plasma potential via the EAE, i.e. by tuning the phase angle $\theta$ between the applied harmonics, is found in both pressure regimes. The profile is almost symmetric for $\theta=45^{\circ}$. It is strongly asymmetric at $\theta=0^{\circ}$ and $\theta=90^{\circ}$ and can be reversed by switching between these two cases. However, the control range, e.g. the difference between the mean plasma potential at $\theta=0^{\circ}$ and $\theta=90^{\circ}$, is bigger at low pressure. This will be explained below.

From these potential profiles, it is obvious that a dc self-bias develops in a geometrically symmetric discharge depending on the choice of $\theta$. The dc self-bias resulting from experiment, simulation, and model is shown in Fig. 7. For the analytical model, the symmetry parameter $\varepsilon$ obtained from the PIC/MCC simulation is used as input parameter. Despite the small deviation at high pressure, which is due to the model assumption of a negligible voltage drop across the plasma bulk, this gives an excellent 

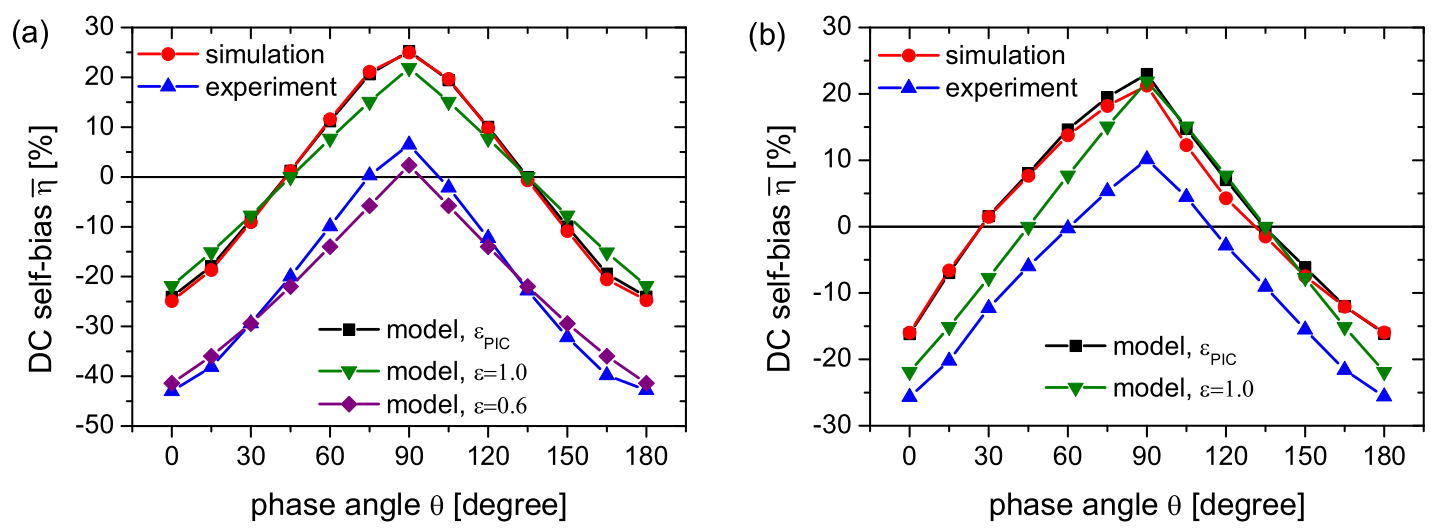

Figure 7. Normalized dc self-bias as a function of the phase angle $\theta$ resulting from experiment, simulation, and model. (a) low pressure (10 Pa), (b) high pressure (100 $\mathrm{Pa}) .\left(\phi_{0}=150 \mathrm{~V}, \mathrm{~d}=2.5 \mathrm{~cm}\right)$

agreement. Similar to the results obtained in argon discharges $[25,27,30]$, the dc selfbias changes almost linearly as a function of the phase angle in the range $0^{\circ} \leq \theta \leq 90^{\circ}$ and $90^{\circ} \leq \theta \leq 180^{\circ}$. In this work, the EAE is tested experimentally for the first time in an electronegative discharge. Due to the controllability of the dc self-bias we find that the effect works in the oxygen scenario investigated here, as well.

However, the control range, i.e. the difference between the minimum possible and maximum possible bias, is smaller at $100 \mathrm{~Pa}$ (Fig. 7 (b)) if compared to the argon case. This is indicated by the comparison of the results in oxygen with the dc self-bias obtained by the analytical model assuming $\varepsilon=1$ for all $\theta$; it corresponds to the assumption of equal mean ion densities in both the powered and the grounded electrode sheath (see Eq. 5), which is well justified in geometrically symmetric argon discharges operated at relatively high pressures $[25,27]$. It should be noted that the experimentally obtained values of the dc self-bias are shifted towards smaller values due to the capacitive coupling between the glass cylinder confining the plasma radially and the outer chamber wall. This effectively enhances the grounded surface area and, thereby, reduces the geometrical symmetry. However, in all cases the dc self-bias changes almost linearly with the phase angle in the range $0^{\circ} \leq \theta \leq 90^{\circ}$ and $90^{\circ} \leq \theta \leq 180^{\circ}$, respectively. It has been shown before and can be confirmed by the data shown in Fig. 6, that the time averaged voltage of the powered and grounded electrode sheath vary linearly with $\bar{\eta}$ [32]. This, in turn, is the most important parameter for the energy of the ions hitting the respective surface. Therefore, the control of $\bar{\eta}$ with the phase angle shown in Fig. 7 is the key to control the ion energy separately from the ion flux via the EAE, which has been examined in argon [24-27] and oxygen [36] discharges up to now.

The effect of a decreased control range of the dc self-bias in oxygen discharges at high pressures can be explained by the density profiles discussed above (see Sec. 3.1). At $100 \mathrm{~Pa}$, the density profile of positive ions has two maxima, which are in the vicinity 
(a)

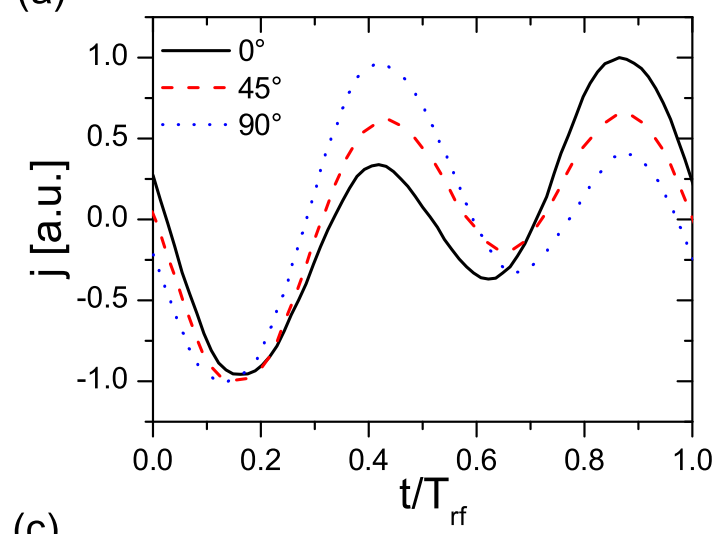

(c)

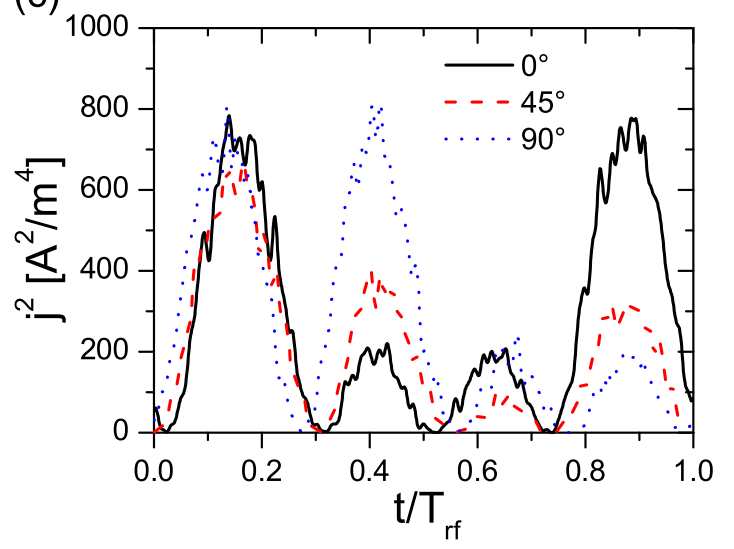

(b)

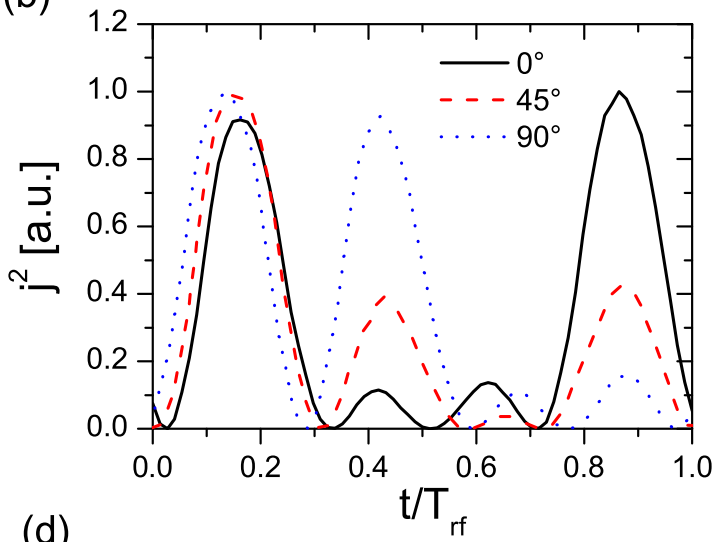

(d)

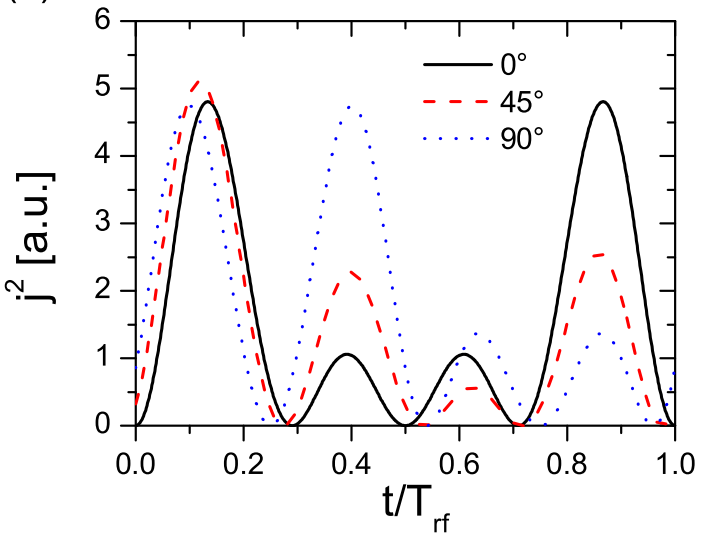

Figure 8. (a) Experimentally determined discharge current as a function of time within one low frequency period for different phase angles at high pressure. The squared current resulting from (b) experiment, (c) simulation, and (d) model is a measure for the power absorbed by electrons. $\left(100 \mathrm{~Pa}, \phi_{0}=150 \mathrm{~V}, \mathrm{~d}=2.5 \mathrm{~cm}\right)$

of the powered and grounded electrode sheath edges, since the production of ions by collisions of fast electrons and neutrals is strongly localized. By diffusion, $\mathrm{O}_{2}^{+}$ions enter the sheath regions, where they are accelerated towards the adjacent electrode. Therefore, the ion density in each of the sheaths depends on the respective peak density of positive ions. Similar to the dynamics in electrically asymmetric argon discharges at relatively high pressures [33], the number of highly energetic electrons is increased at $\theta=45^{\circ}$ and $\theta=135^{\circ}$ at the powered and grounded electrode, respectively.

This can be examined in more detail by analyzing the time dependent electron conducting current. Electron heating at the powered and grounded electrode sheath [52-55, 65-77] leads to a negative and positive current, respectively. Figure 8 (a) shows the measured discharge current as a function of time within one low frequency period at $100 \mathrm{~Pa}$. The squared current resulting from (b) experiment, (c) simulation, and (d) model is a measure for the power absorbed by electrons. In the model, the charge voltage balance (Eq. 2) without the bulk contribution is solved for $q(t)$. The normalized current is found by differentiation. Here, the experimentally determined dc self-bias, $\bar{\eta}$, and 
symmetry parameter, $\varepsilon$, are used as input parameters. We find an excellent agreement between experiment, simulation, and model. At $\theta=0^{\circ}$ and $\theta=90^{\circ}$, the current shows a symmetric behavior: its shape basically consists of a strong and a weak maximum and a strong and a weak minimum, respectively (see Fig. 8 (a)). Therefore, electrons are heated mainly at two times within the rf period, once at the powered electrode sheath and once at the grounded electrode sheath (see Fig. 8 (b)-(d)). Consequently, they gain about the same amount of energy at both sheaths during one low frequency period. At $\theta=45^{\circ}$, one strong minimum and two relatively small maxima are found in the discharge current. This means, that the electron heating is stronger at the powered electrode, even though the time of power dissipation is shorter (see Fig. 8 (b)-(d)). Accordingly, electron heating, ionization, and the peak density of $\mathrm{O}_{2}^{+}$are enhanced in front of the grounded electrode and $\varepsilon>1$ is found. At $\theta=135^{\circ}$, the symmetry of the discharge is reversed, i.e. the sign and direction of the current is reversed. Therefore, the major gain of electron energy takes place at the grounded electrode sheath. This asymmetry of the electron heating at $\theta=45^{\circ}$ and $\theta=135^{\circ}$ leads to a higher ionization rate and, by that, to a higher peak density on the corresponding side. The resulting modulation of the sheath densities as a function of $\theta$, which also affects the symmetry parameter, in turn changes the electron heating by sheath expansion.

Altogether, this effect leads to a phase angle dependence of the symmetry parameter, which is in contrast to the constancy of $\varepsilon \approx 1$ obtained in an argon discharge operated under similar conditions [25]. The change of $\varepsilon(\theta)$ causes smaller absolute values of the de self-bias $\bar{\eta}$ (see Eq. 3) and, thus, a damping of $\bar{\eta}$ as a function of $\theta$ in oxygen at high pressures. It also explains the small curvatures of $\bar{\eta}(\theta)$ shown in Fig. 7 (b). This correlation is also illustrated by the comparison of $\varepsilon$ with the ratio of the peak density of positive ions in front of the powered $\left(\mathrm{O}_{2}^{+, p}\right)$ and grounded $\left(\mathrm{O}_{2}^{+, g}\right)$ electrode depicted in Fig. 9 (b). The experimentally determined symmetry parameter qualitatively shows the same behavior. However, it always is smaller than $\varepsilon$ from PIC/MCC simulations. Again, this is caused by the nonideal geometrical symmetry. We find in the experiment that, under the conditions investigated here, $\bar{\eta}$ and $\varepsilon$ are stable, even if the gas flow is varied or if we change from a pure oxygen discharge to a gas composition of oxygen and argon by admixing different amounts of Ar gas.

At low pressure, the symmetry parameter changes only by about $\pm 8 \%$ (see Fig. 9 ). Here, it is increasing as a function of the phase angle in the range $0^{\circ} \leq \theta \leq 90^{\circ}$ and decreasing in the range $90^{\circ} \leq \theta \leq 180^{\circ}$ (see Fig. 9 (a)). The difference between the mean sheath voltages, i.e. the occurence of a dc self-bias, affects the mean ion densities in the sheaths. This leads to a small self-amplification of the dc self-bias generated via the EAE [24, 25] and, accordingly, a slightly bigger control range in both experiment and simulation compared to the assumption of a constant $\varepsilon$ (see Fig. 7 (a)). Here, it can also be seen that a symmetry parameter $\varepsilon=$ const. different from unity only leads to a shift of the bias curve towards smaller $(\varepsilon<1)$ or higher $(\varepsilon>1)$ values. 

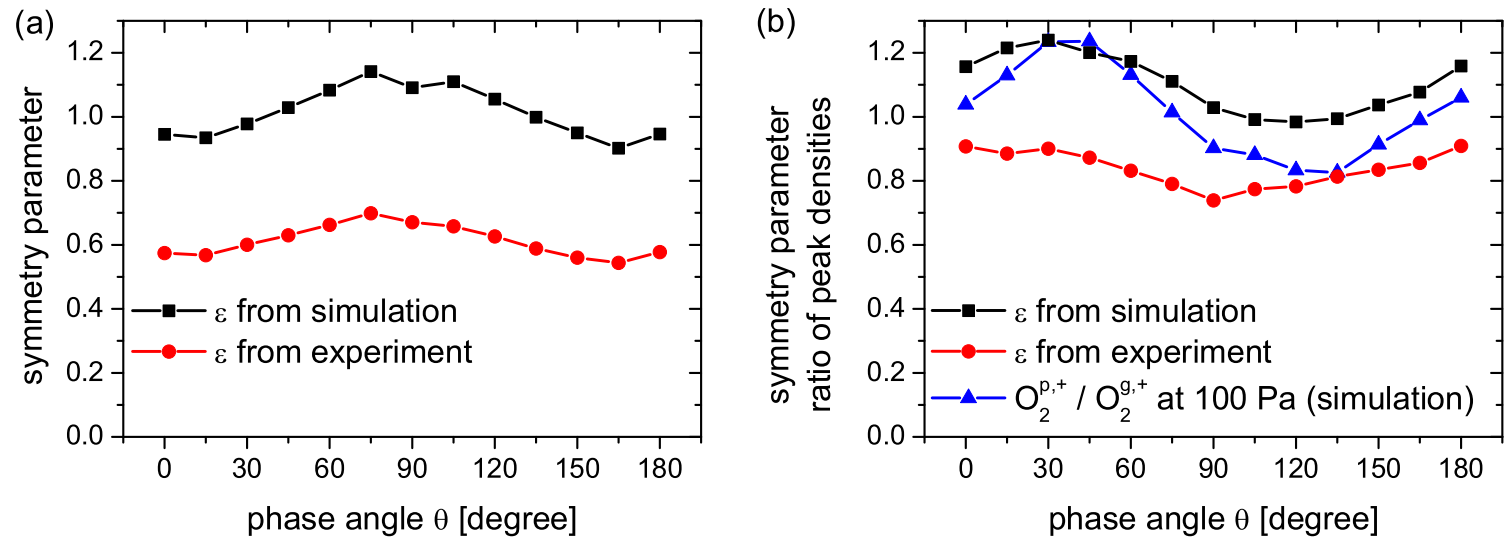

Figure 9. Symmetry parameter $\varepsilon$ as a function of the phase angle $\theta$ resulting from $\mathrm{PIC} / \mathrm{MCC}$ simulations and experiment at (a) $10 \mathrm{~Pa}$ and (b) $100 \mathrm{~Pa}$. The ratio of the peak density of positive ions in front of the powered $\left(O_{2}^{+, p}\right)$ and grounded $\left(\mathrm{O}_{2}^{+, g}\right)$ electrode obtained from the simulation at high pressure $(100 \mathrm{~Pa})$ is also shown. $\left(\phi_{0}=150 \mathrm{~V}, \mathrm{~d}=2.5 \mathrm{~cm}\right)$

\section{3. control of the self-excitation of PSR oscillations}

The self-excitation of the plasma series resonance (PSR) is an important issue in asymmetric capacitive discharges operated at low pressures, because it enhances the electron heating $[65,67,73-76,86-92]$. Due to Eq. 2, the nonlinearities of the plasma sheaths do not cancel if $\varepsilon \neq 1$ and resonance oscillations occur. In general, it does not matter whether this asymmetry is induced geometrically $\left(A_{p} \neq A_{g}\right)$ or electrically $\left(\phi_{\sim, \max } \neq-\phi_{\sim, \min }\right)[28]$. It should be noted that the discharge symmetry is characterized by $\varepsilon$ and not by the value of $\bar{\eta}$ [37]; even though we could define an electrical symmetry by $\bar{\eta}=0$, this would not necessarily mean that the densities are symmetric with respect to the discharge center, which would imply $\varepsilon=1$ in the case of a geometrically symmetric discharge.

Figure 10(a)-(c) shows the time resolved discharge current within one low frequency period for different phase angles at low pressure resulting from experiment, simulation, and model. In the model, the PSR can be examined if the voltage drop across the plasma bulk is taken into account, i.e. by solving the charge voltage balance (Eq. 2) for $q(t)$ and calculating $\dot{q}(t)$ [34]. We choose the input parameters of the model according to the experimental results. Taking the values of $\bar{\eta}(\theta)$ obtained from the experiment (see Fig. 7 (a)) allows us to calculate the symmetry parameter, $\varepsilon$, (see Fig. 9 (a)) and the total charge, $q_{t}$, using Eqs. 3 and 4. At $10 \mathrm{~Pa}$, the electron momentum changing collision frequency can be estimated $\nu_{m} \approx 1.2 \cdot 10^{8} \mathrm{~s}^{-1}$. From the optical appearance of the plasma, we assume $L_{b} \approx 1.0 \mathrm{~cm}$ and $s_{\max , p} \approx 0.5 \mathrm{~cm}$, which is in satisfying agreement with the PIC/MCC simulation data shown above. We also roughly estimate the mean electron density in the plasma bulk to be $5 \cdot 10^{8} \mathrm{~cm}^{-3}$, which might be reasonable due to the simulated density profiles shown in Fig. 1. 

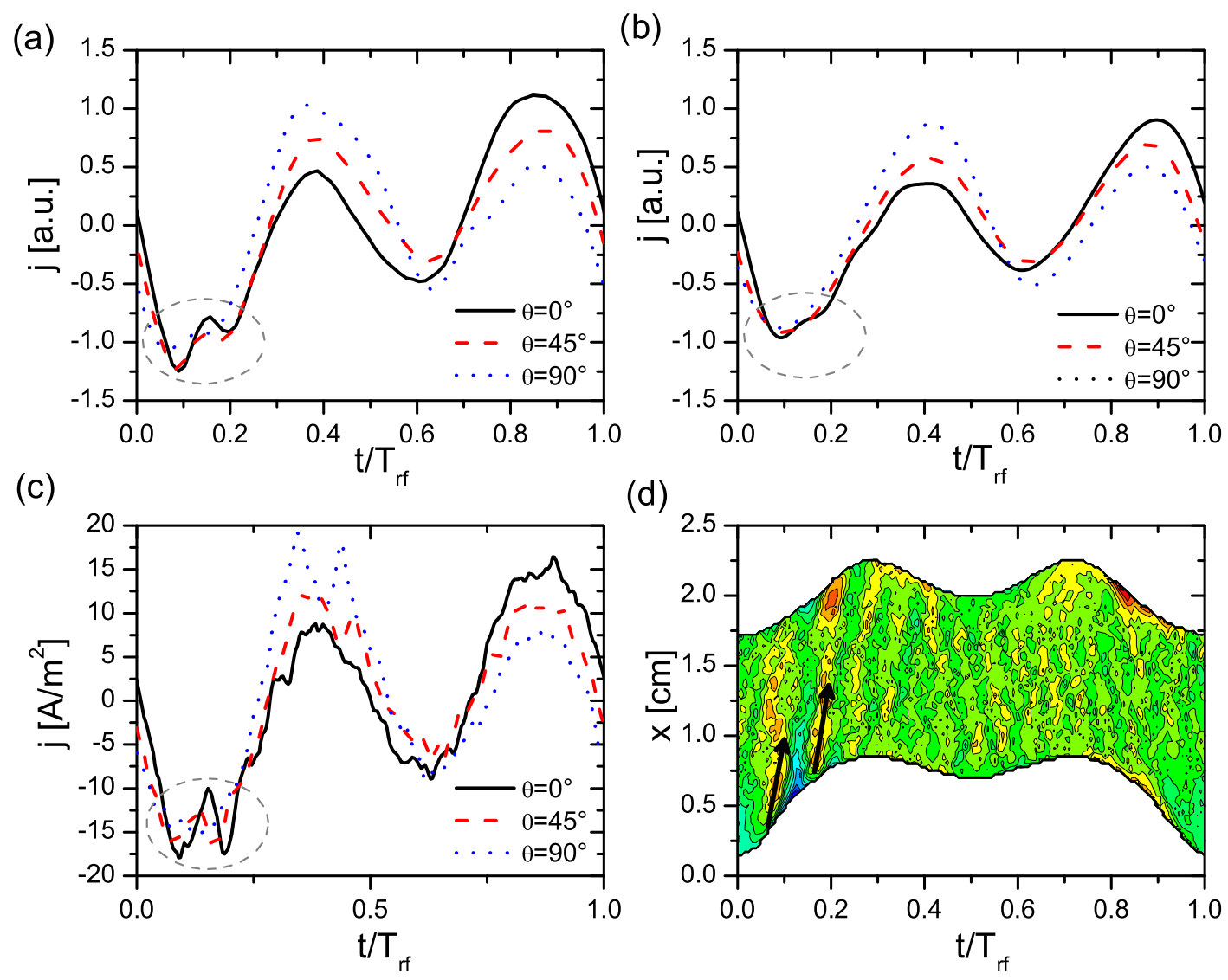

(d)

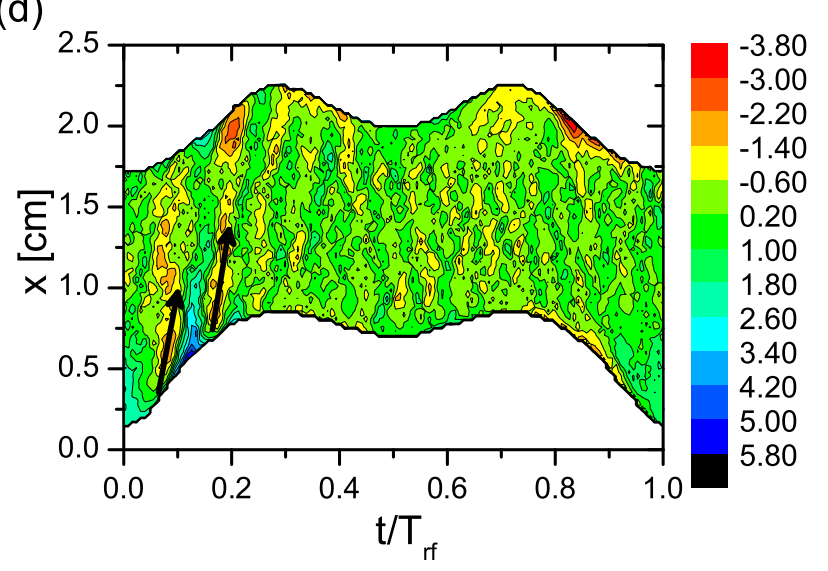

Figure 10. (a)-(c) Discharge current as a function of time within one low frequency period for different phase angles at low pressure resulting from (a) experiment, (b) model, and (c) simulation. The input parameters of the model are chosen according to the experimental data and are described in the text. (d) Spatially resolved displacement current in the plasma bulk region within one low frequency period for the case of $\theta=0^{\circ}$ resulting from the simulation. The powered electrode is located at $\mathrm{x}=0$. The color scale gives the displacement current density in $\left[A / \mathrm{m}^{2}\right] .\left(10 \mathrm{~Pa}, \phi_{0}=150 \mathrm{~V}\right.$, $\mathrm{d}=2.5 \mathrm{~cm})$

At the time of maximum or minimum discharge current (corresponding to the time of the collapse of one of the sheaths), oscillations with a small amplitude, but afrequency of about one order of magnitude higher than the applied fundamental frequency are self-excited in experiment, simulation, and model. Of course, the amplitude of these PSR oscillations is very small compared to the total current amplitude due to the relatively small asymmetry. This is different from the "classical" scenario of the PSR self-excited in geometrically asymmetric discharges [65, 86-91], where usually $\varepsilon \ll 1$ and NERH (Nonlinear Electron Resonance Heating) gives a large contribution in the electron heating $[65,87,92]$. Due to the symmetry parameter $\varepsilon$ shown in Fig. $9, \varepsilon \approx 1$ at $10 \mathrm{~Pa}$ and $\theta=45^{\circ}$ in the PIC/MCC simulation and the PSR almost vanishes in this case. Therefore, the small amplitudes of the PSR oscillations in the discharge current can be controlled in a geometrically symmetric electrically asymmetric capacitively 
coupled oxygen discharge. Accordingly, NERH can be neglected. The electrically induced asymmetry of the discharge (see Fig. 9 (a)) is just too small to cause a reasonably enhanced electron heating. This explains, why the time averaged electron power absorption is independent of $\theta$ even at low pressures as discussed above (see Fig. $5(\mathrm{a}))$.

In contrast, in the electron current obtained in the experiment and the model using experimentally determined values, the PSR oscillations are strongest at $\theta=0^{\circ}$ and become smaller with increasing $\theta$. Again, this is caused by the symmetry parameter $\varepsilon$ changing as a function of the phase angle. Here, $\varepsilon$ is smaller than unity, but increases with $\theta$ (see Fig. $9(\mathrm{a})$ ). Therefore, the discharge becomes more symmetric by tuning $\theta$ from $0^{\circ}$ to $90^{\circ}$, because the small geometrical asymmetry found in the experiment is partly compensated by the electrical asymmetry.

However, the amplitude of the high frequency oscillations at $\theta=0^{\circ}$ is distinctly smaller in the model compared to the results of experiment and simulation. The reason for this deviation is most likely caused by the low electron density in the plasma bulk (see Fig. 1(a)). This allows for resonance oscillations at the plasma frequency, which in electropositive discharges typically is well above the frequency of the PSR oscillations $\left(\omega_{P S R} \approx \sqrt{s_{\max , p} / L_{b}} \omega_{p, e}[87]\right)$. According to the simulation data discussed in Sec. 3.1 (see Fig. 1), these frequencies can be estimated $\omega_{P S R} \approx 0.98 \cdot 10^{9} \mathrm{~s}^{-1}<\omega_{p, e} \approx$ $1.13 \cdot 10^{9} \mathrm{~s}^{-1}$. Therefore, both frequencies are very close.

The PSR oscillations lead to an enhanced acceleration of the sheath expansion velocity, which results in the generation of electron beams with very high velocities if compared to the thermal velocity of the bulk electrons. The current density of this beam is $\left|\vec{j}_{\text {beam }}\right|=e n_{\text {beam }}\left|\vec{u}_{\text {beam }}\right|$. Under the assumption of a quasineutral sheath region at the time of sheath collapse, the beam electron density is about $1.5 \cdot 10^{13} \mathrm{~m}^{-3}$ according to the ion density profile shown in figure 1(a). In a rough estimation, the beam velocity has a value of about $4 \cdot 10^{6} \mathrm{~m} \mathrm{~s}^{-1}$. As will be understood later, this can also be found in the tilting of the arrows shown in figure $10(\mathrm{~d})$. This yields a current density $\left|\vec{j}_{\text {beam }}\right|$ of the order of $10 \mathrm{Am}^{-2}$, which is in reasonable agreement with the current density depicted in figure $10(\mathrm{c})$.

When these beam electrons enter the bulk they cause a rather strong electric field. This is because the electron density in the plasma bulk and, accordingly, the bulk conductivity, $\sigma_{b}$, are low [1]. With the electron density given in figure 1(a), we find $\sigma_{b} \approx 140 \cdot 10^{-3} \Omega^{-1}$. Therefore, the electric field strength $\left|\vec{E}_{\text {beam }}\right|=\left|\vec{j}_{\text {beam }}\right| / \sigma_{b}$ has to be of the order of $100 \mathrm{Vm}^{-1}$. In a very simple picture, this field is completely shielded by the bulk electrons after one plasma frequency. Then, the resulting displacement current inside the plasma bulk should be of the order of $\varepsilon_{0}\left|\vec{E}_{\text {beam }}\right| \cdot \omega_{p, e} \approx 1 \mathrm{Am}^{-2}$. In other words, the beam current is distributed in Fourier space around the PSR frequency. As discussed above, the PSR frequency is very close to the electron plasma frequency in our case. Therefore, a reasonable part of the current has a frequency higher than $\omega_{p, e}$, which can excite an electrostatic wave $[67,93]$ accompanied by a displacement current. 
This rough estimation shows that the displacement current within the plasma bulk is not negligible in an oxygen discharge under the low pressure conditions investigated here.

Figure 10(d) shows the displacement current density in the plasma bulk region at $\theta=0^{\circ}$. The upper and lower boundaries of the colour plot are choosen to suppress the strong displacement current in the sheaths. However, they do not correspond to the exact sheath motion. As can be verified here, the contribution of the displacement current to the total current is not negligible at the time of the expansion of the sheath in front of the powered electrode, i.e. the time of strongly pronounced PSR oscillations. The amplitude of the displacement current is in reasonable agreement with the above estimation. This is not taken into account in the analytical model. Therefore, the model assumption of a pure conduction current in the plasma bulk underestimates the strength of the resonance. Even though the amplitude of the resonance oscillations is smaller in the model for this reason, a damping of it can be observed at higher $\theta$ in Fig. 10(b).

This double resonance structure might be of great importance for all asymmetric electronegative discharges operated at low pressures, since the requirement of a rather low electron density in the plasma bulk is mostly fulfilled. Both resonances are governed by the second derivative of the charge $q$ with respect to time, $\ddot{q}(t)$, occuring in the plasma bulk term (see Eq. 2) [87]. This is directly proportional to $\ddot{\phi}_{\sim}(t)$. At $\theta=0^{\circ}$, the absolute value of $\ddot{\phi}_{\sim}(t)$ is maximum at the beginning of the low frequency period. Therefore, we find a strong impact of both resonances in the first part of the rf cycle. It might be possible to model these results accurately. However, the coupling certainly needs a more detailed treatment, which should be done in a future work.

\section{Conclusions}

The Electrical Asymmetry Effect has been investigated in a dual-frequency capacitively coupled radio-frequency discharge in pure oxygen. Our results show that many of plasma parameters can be controlled via the EAE by tuning the phase angle $\theta$ between the applied harmonics. In particular, it is observed that the density profiles of all charged species are shifted between the two electrodes due to the dependence of the sheath extensions on $\theta$, while the total densities stay rather constant, varying only within $\pm 10 \%$ for different $\theta$. This is because the time averaged power absortption of the electrons does not vary much with $\theta$. The bulk length is found to change slightly with $\theta$, leading to small modulations in the densities of electrons and ions and consequently the electronegativity of the oxygen plasma.

The time averaged potential profiles are found to be dependent closely on $\theta$, as the voltage drop across both sheaths can be varied via the symmetry of the applied voltage waveform. The dc self-bias is approximately a linear function of $\theta$. The variation range of the dc self-bias is smaller at relatively high pressures, due to the localized ionizations in the sheath regions at high pressures. This leads to a higher positive ion density in the sheath, and an enhanced electron heating therein. This mechanism might 
become more important in electrically asymmetric discharges, which are operated in other (electronegative) gases at even higher pressures.

At low pressures, the plasma series resonance is self-excited in asymmetric discharges resulting in high frequency oscillations in the temporal evolution of the conducting current, though only small amplitude PRS oscillations are observed in comparison to those typically generated in geometrically asymmetric discharges. Nevertheless, a certain amount of resonance oscillations and consequently the Nonlinear Electron Resonance Heating can be switched on and off by tuning $\theta$, i.e. by electrically controlling the discharge symmetry. In addition, these PSR oscillations are superimposed by another resonance structure, i.e. an electrostatic wave close to the electron plasma frequency due to the low electron density in the bulk region. This can be regarded as a principal mechanism in low pressure electronegative discharges.

In conclusion, the EAE has been verified experimentally for the first time in an electronegative discharge. And we show that it is an effective way to control many plasma paramters by using the EAE in electronegative discharges. Especially, we examine the underlying mechanisms to realize the separate control of the ion flux and ion bombarding energy onto the electrodes. Although further investigations, e.g. in other gases, are needed to explore the applicability of the EAE in a broader scope of CCRF discharges, this work clearly motivates applying this effect to strongly electronegative plasmas, such as dusty plasmas.

\section{Acknowledgments}

Funding by the German Federal Ministry for the Environment, Nature conservation, and Nuclear Safety (0325210B). The support by the Hungarian Fund for Scientific Research (OTKA K77653 and OTKA IN-85261), the Alexander von Humboldt Foundation, and the Ruhr-University Research Department Plasma is gratefully acknowledged. This work was supported by the National Natural Science Foundation of China (Grant No. 10635010) and the Research Fund for Doctoral Program of Higher Education of China (Grant No. 20090041110026).

\section{References}

[1] Lieberman M A and Lichtenberg A J 2005 Principles of Plasma Discharges and Materials Processing Wiley, New York (2nd ed.)

[2] Schulze J 2009 PhD thesis, Ruhr University Bochum, Germany http://wwwbrs.ub.ruhr-unibochum.de/netahtml/HSS/Diss/SchulzeFelixJulian/diss.pdf

[3] Boyle P C, Ellingboe A R and Turner M M 2004 Plasma Sources Sci. Technol. 13 493

[4] Boyle P C, Ellingboe A R and Turner M M 2004 J. Phys. D: Appl. Phys. 37697

[5] Kitajima T, Takeo Y, Petrovic Z L and Makabe T 2000 Appl. Phys. Lett. 77489 
[6] Denda T, Miyoshi Y, Komukai Y, Goto T, Petrovic Z L and Makabe T $2004 \mathrm{~J}$. Appl. Phys. 95870

[7] Lee J K, Manuilenko O V, Babaeva N Yu, Kim H C and Shon J W 2005 Plasma Sources Sci. Technol. 1489

[8] Booth J P, Curley G, Marić D and Chabert P 2010 Plasma Sources Sci. Technol. 19015005

[9] Gans T, Schulze J, O'Connell D, Czarnetzki U, Faulkner R, Ellingboe A R and Turner M M 2006 Appl. Phys. Lett. 89261502

[10] Schulze J, Gans T, O'Connell D, Czarnetzki U, Ellingboe A R and Turner M M 2007 J. Phys. D: Appl. Phys. 407008

[11] Schulze J, Donkó Z, Luggenhölscher D and Czarnetzki U 2009 Plasma Sources Sci. Technol. 18034011

[12] Turner M M and Chabert P 2006 Phys. Rev. Lett. 96261502

[13] Donkó Z, 2007 Proc. of Symposium of Application of Plasma Processes, Slovakia

[14] Ahn S K and Chang H Y 2009 Appl. Phys. Lett. 95111502

[15] Olevanov M, Proshina O, Rakhimova T and Voloshin D 2008 Phys. Rev. E 78 026404

[16] Li X S, Bi Z H, Chang D L, Li Z C, Wang S, Xu X, Xu Y, Lu W Q, Zhu A M and Wang Y N 2008 Appl. Phys. Lett. 93031504

[17] Wang S, Xu X and Wang Y N 2007 Phys. Plasmas 14113501

[18] Georgieva V and Bogaerts A 2006 Plasma Sources Sci. Technol. 15368

[19] Yang Y and Kushner M J 2010 J. Appl. Phys. 108113306

[20] Schulze J, Donkó Z, Luggenhölscher D and Czarnetzki U 2009 Plasma Sources Sci. Technol. 18034011

[21] Waskoenig J and Gans T 2010 Appl. Phys. Lett. 96181501

[22] Donkó Z, Schulze J, Hartmann P, Korolov I, Czarnetzki U and Schüngel E 2010 Appl. Phys. Lett. 97081501

[23] Heil B G, Schulze J, Mussenbrock T, Brinkmann R P and Czarnetzki U 2008 IEEE Trans. on Plasma Sci. 361404

[24] Heil B G, Czarnetzki U, Brinkmann R P and Mussenbrock T 2008 J. Phys. D: Appl. Phys. 41165202

[25] Donkó Z, Schulze J, Heil B G and Czarnetzki U 2009 J. Phys. D: Appl. Phys. 42 025205

[26] Czarnetzki U, Heil B G, Schulze J, Donko Z, Mussenbrock T and Brinkmann R P 2009 IOP Conf. Series 162012010

[27] Schulze J, Schüngel E, Donkó Z and Czarnetzki U 2009 J. Phys. D FTC 42092005

[28] Donkó Z, Schulze J, Czarnetzki U and Luggenhölscher D 2009 Appl. Phys. Lett. 94 131501 
[29] Longo S and Diomede P 2009 Plasma Processes and Polymers 6370

[30] Schulze J, Schüngel E, Donkó Z and Czarnetzki U 2009 J. Appl. Phys. 106063307

[31] Schulze J, Schüngel E, Donkó Z and Czarnetzki U 2010 J. Phys. D 43225201

[32] Schüngel E, Schulze J, Donkó Z and Czarnetzki U 2011 Phys. Plasmas 18013503

[33] Schulze J, Schüngel E, Donkó Z and Czarnetzki U 2010 Plasma Sources Sci. Technol. 19045028

[34] Czarnetzki U, Schulze J, Schüngel E and Donkó Z 2011 Plasma Sources Sci. Technol. accepted for publication

[35] Johnson E V, Verbeke T, Vanel J-C and Booth J-P 2010 J. Phys. D: Appl. Phys. 43412001

[36] Zhang Q-Z, Jiang W, Hou L-J and Wang Y-N 2011 J. Appl. Phys. 109013308

[37] Schulze J, Schüngel E, Czarnetzki U, Gebhardt M, Brinkmann R P and Mussenbrock T 2011 Appl. Phys. Lett. 98031501

[38] Mogab C J, Adams A C and Flamm D L 1978 J. Appl. Phys. 493796

[39] d'Agostino R, Cramarossa F, De Benedictis S and Ferraro G 1981 J. Appl. Phys. 521259

[40] Kushner M J 1993 J. Appl. Phys. 746538

[41] Berezhnoj S V, Shin C B, Buddemeier U and Kaganovich I 2000 Appl. Phys. Lett. 77800

[42] Lichtenberg A J, Vahedi V, Lieberman M A and Rognlien T 1994 J. Appl. Phys. 752339

[43] Kaganovich I Phys. Plasmas 200182540

[44] Bogdanov E A, Kolobov V I, Kudryavtsev A A and Tsendin L D 2002 Technical Physics 47946

[45] Gudmundsson J T 2004 J. Phys. D: Appl. Phys. 372073

[46] Gudmundsson J T, Kouznetsov I G, Patel K K and Lieberman M A 2001 J. Phys. D: Appl. Phys. 341100

[47] Franklin R N and Snell J 1999 J. Phys. D: Appl. Phys. 322190

[48] Bronold F X, Matyash K, Tskhakaya D, Schneider R and Fehske H 2007 J. Phys. D: Appl. Phys. 406583

[49] Vender D, Stoffels W W, Stoffels E, Kroesen G M W and de Hoog F J 1995 Phys. Rev. E 512436

[50] Stoffels E, Stoffels W W, Vender D, Kando M, Kroesen G M W and de Hoog F J 1995 Phys. Rev. E 512425

[51] Kaga K, Kimura T and Ohe K 2001 Jpn. J. Appl. Phys. 40330

[52] Matyash K, Schneider R, Dittmann K, Meichsner J, Bronold F X and Tskhakaya D 2007 J. Phys. D: Appl. Phys. 406601 
[53] Dittmann K, Drozdov D, Krames B and Meichsner J 2007 J. Phys. D: Appl. Phys. 406593

[54] Nemschokmichal S, Dittmann K and Meichsner J 2008 IEEE Trans. on Plasma Sci. 361360

[55] Küllig C, Dittmann K and Meichsner J 2010 Plasma Sources Sci. Technol. 19 065011

[56] Katsch H M, Tewes A, Quandt E, Goehlich A, Kawetzki T and Döbele H F 2000 J. Appl. Phys. 886232

[57] Meichsner J, Zeuner M, Krames B, Nitschke M, Rochotzki R and Barucki K 1998 Surf. Coat. Technol. 981565

[58] Coburn J W and Kay E 1972 J. Appl. Phys. 434965

[59] Birdsall C K and Langdon A B 1985, Plasma Physics via Computer Simulation (McGraw-Hill, New York).

[60] Yonemura S and Nanbu K 2003, IEEE Trans. Plasma Sci. 31, 479

[61] Vahedi V, Surendra M 1995, Comput. Phys. Commun. 87179

[62] Denpoh K and Nanbu K 1998, J. Vac. Sci. Technol. A 161201

[63] Kawamura E 2003, Particle-in-Cell simulations of bounded plasma discharges applied to low pressure high density sources and positive columns. Ph.D. thesis, University of California

[64] Brinkmann R P 2007 J. Appl. Phys. 102093303

[65] Ziegler D, Trieschmann J, Mussenbrock T, Brinkmann R P, Schulze J, Czarnetzki U, Semmler E, Awakowicz P, O'Connel D and Gans T 2010 Plasma Sources Sci. Technol. 19045001

[66] Belenguer P and Boeuf J P 1990 Phys. Rev. A 414447

[67] Vender D and Boswell R W 1992 J. Vac. Sci. Tech. A 101331

[68] Park G Y and Lee J K 2008 J. Phys. D: Appl. Phys. 41022004

[69] Mahony C M O and Graham W G 1999 IEEE Trans. on Plasma Sci. 2772

[70] Gans T, Lin C C, Schulz-von der Gathen V and Döbele H F 2003 Phys. Rev. A 67 012707

[71] Tochikubo F, Suzuki A, Kakuta S, Terazono Y and Makabe T 1990 J. Appl. Phys. 685532

[72] Wood B P 1991 Sheath heating in low pressure capacitive radio frequency discharges PhD Thesis University of California at Berkeley

[73] Schulze J, Kampschulte T, Luggenhölscher D and Czarnetzki U 2007 J. Phys. Conf. Ser. 86012010

[74] Schulze J, Heil B G, Luggenhölscher D, Brinkmann R P and Czarnetzki U 2008 J. Phys. D. 41195212 
[75] Schulze J, Heil B G, Luggenhölscher D, Mussenbrock T, Brinkmann R P and Czarnetzki U 2008 J. Phys. D. FTC 41042003

[76] Schulze J, Heil B G, Luggenhölscher D and Czarnetzki U 2008 IEEE Tran. on Plasma Sci. 361400

[77] Rauf S, Bera K and Collins K 2010 Plasma Sources Sci. Technol. 19015014

[78] Shibata M, Nakano N and Makabe T 1995 J. Appl. Phys. 776181

[79] Shibata M, Makabe T and Nakano N 1998 Jpn. J. Appl. Phys. 374182

[80] Lisovskiy V A and Yegorenkov V D 2006 Vacuum 80458

[81] Nakano N and Makabe T 1995 J. Phys. D: Appl. Phys. 2831

[82] Lisovskiy V, Booth J P, Jolly J, Martins S, Landry K, Douai D, Cassagne V and Yegorenkov V 2007 J. Phys. D: Appl. Phys. 406989

[83] Aoyagi K, Hirose Y, Ishikawa I, Saito Y and Suganomata S 1997 Jpn. J. Appl. Phys. 365290

[84] Gottscho R A 1987 Phys. Rev. A 362233

[85] Lisovskiy V A and Yegorenkov V D 2004 Vacuum 7419

[86] Mussenbrock T and Brinkmann R P 2007 Plasma Sources Sci. Technol. 16377

[87] Czarnetzki U, Mussenbrock T and Brinkmann R P 2006 Phys. Plasmas 13123503

[88] Mussenbrock T, Brinkmann R P, Lieberman M A, Lichtenberg A J and Kawamura E 2008 Phys. Rev. Lett. 101085004

[89] Ziegler D, Mussenbrock T and Brinkmann R P 2008 Plasma Sourc. Sci. Technol. 17045011

[90] Ziegler D, Mussenbrock T and Brinkmann R P 2009 Phys. Plasmas 16023503

[91] Klick M 1996 J. Appl. Phys. 793445

[92] Yamazawa Y 2009 Appl. Phys. Lett. 95191504

[93] O'Connell D, Gans T, Vender D, Czarnetzki U, Boswell R 2007 Phys. Plasmas 14 034505 